\title{
Z-Spec: a broadband millimeter-wave grating spectrometer: design, construction, and first cryogenic measurements
}

C. Matt Bradford, Peter A. R. Ade, James E. Aguirre, James J. Bock, Mark Dragovan, et al.

C. Matt Bradford, Peter A. R. Ade, James E. Aguirre, James J. Bock, Mark Dragovan, Lionel Duband, Lieko Earle, Jason Glenn, Hideo Matsuhara, Bret J. Naylor, Hien T. Nguyen, Minhee Yun, Jonas Zmuidzinas, "Z-Spec: a broadband millimeter-wave grating spectrometer: design, construction, and first cryogenic measurements," Proc. SPIE 5498, Millimeter and Submillimeter Detectors for Astronomy II, (8 October 2004); doi: 10.1117/12.552182

Event: SPIE Astronomical Telescopes + Instrumentation, 2004, Glasgow, United Kingdom 


\title{
Z-Spec: a broadband millimeter-wave grating spectrometer -- design, construction, and first cryogenic measurements
}

\author{
C.M. Bradford ${ }^{1}$, P. Ade ${ }^{2}$, J. Aguirre ${ }^{3}$, J.J. Bock ${ }^{1}$, M. Dragovan ${ }^{1}$, L. Duband ${ }^{4}$, \\ L. Earle ${ }^{3}$, J. Glenn $^{3}$, H. Matsuhara ${ }^{5}$, B.J. Naylor ${ }^{6}$, H. Nguyen ${ }^{1}$, M. Yun ${ }^{1}$, J. Zmuidzinas ${ }^{6}$ \\ ${ }^{1}$ Jet Propulsion Laboratory, Caltech, Pasadena, CA, \\ ${ }^{2}$ Cardiff University, Cardiff, UK, \\ ${ }^{3}$ CASA, University of Colorado, Boulder, CO, \\ ${ }^{4}$ Commissariat à l'Energie Atomique, Paris, France, \\ ${ }^{5}$ Institute of Space and Astronautical Science, Sagamihara, Japan, \\ ${ }^{6}$ California Institute of Technology, Pasadena, CA
}

\begin{abstract}
We present the design, integration, and first cryogenic testing of our new broad-band millimeter-wave spectrometer, ZSpec. Z-Spec uses a novel architecture called WaFIRS (Waveguide Far-IR Spectrometer), which employs a curved diffraction grating in a parallel-plate waveguide propagation medium. The instrument will provide a resolving power between 200 and 350 across an instantaneous bandwidth of $190-310 \mathrm{GHz}$, all packaged within a cryostat that is of order 1 meter in size. For background-limited astronomical observations in the $1 \mathrm{~mm}$ terrestrial window, Z-Spec uses 160 silicon nitride micro-mesh bolometers and the detectors and waveguide grating are cooled to $\sim 0.1 \mathrm{~K}$. Our first cryogenic meausurements at $225 \mathrm{GHz}$ show resolving power greater than 200, and the end-to-end throughput is estimated to be greater than $30 \%$, possibly as high as $40 \%$. Z-Spec represents the first systematic approach to cosmological redshift measurement that is not based on optical or near-IR identifications. With its good sensitivity and large bandwidth, ZSpec provides a new capability for millimeter-wave astrophysics. The instrument will be capable of measuring rotational carbon monoxide line emission from bright dusty galaxies at redshifts of up to 4, and the broad bandwidth insures that at least two lines will be simultaneously detected, providing an unambiguous redshift determination. In addition to Z-Spec's observations over the next 1-3 years, the WaFIRS spectrometer architecture makes an excellent candidate for mid-IR to millimeter-wave spectrometers on future space-borne and suborbital platforms such as SPICA and SAFIR. The concept is dramatically more compact and lightweight than conventional free-space grating spectrometers, and no mirrors or lenses are used in the instrument. After the progress report on Z-Spec, we highlight this capability.
\end{abstract}

Keywords: millimeter-wave, spectroscopy, bolometers, cryogenic, waveguide, diffraction grating, redshift

\section{INTRODUCTION}

The discovery of galaxies beyond $\mathrm{z} \sim 1$ which emit the bulk of their luminosity at long wavelengths has demonstrated the need for high-sensitivity, broad-band spectroscopy in the far-IR/submm $/ \mathrm{mm}$ bands. Because many of these sources are not detectable in the optical, long-wavelength spectroscopy is key to measuring their redshifts and interstellar medium conditions. The continuum source list will increase in the coming decade as the cosmic far-IR background is resolved into its constituent galaxies with new ground-based instruments (SCUBA2, Bolocam, MAMBO), and the surveys of Herschel and Spitzer. Yet the planned spectroscopic capabilities lag behind, in part due to the difficulty in scaling existing IR spectrograph designs to longer wavelengths. To overcome these limitations, we are developing WaFIRS, a novel concept for long-wavelength spectroscopy which utilizes a parallel-plate waveguide and a curved diffraction grating. Our first prototype forms the heart of the Z-Spec instrument, a cryogenic mm-wave spectrometer we are constructing for the $190-310 \mathrm{GHz}$ terrestrial atmospheric window. Z-Spec will measure redshifts of dusty galaxies using multiple lines in the CO rotational ladder. This paper presents a progress report on Z-Spec, including our first cryogenic measurements. This document refers heavily to two papers from the 2002 SPIE conference

\footnotetext{
${ }^{1}$ For further information contact Matt Bradford, e-mail: bradford@submm.caltech.edu, phone: (818) 393-7499.
} 
which outline the WaFIRS design concept and the philosophy of Z-Spec (B02, N02), as well as the references cited in these works.

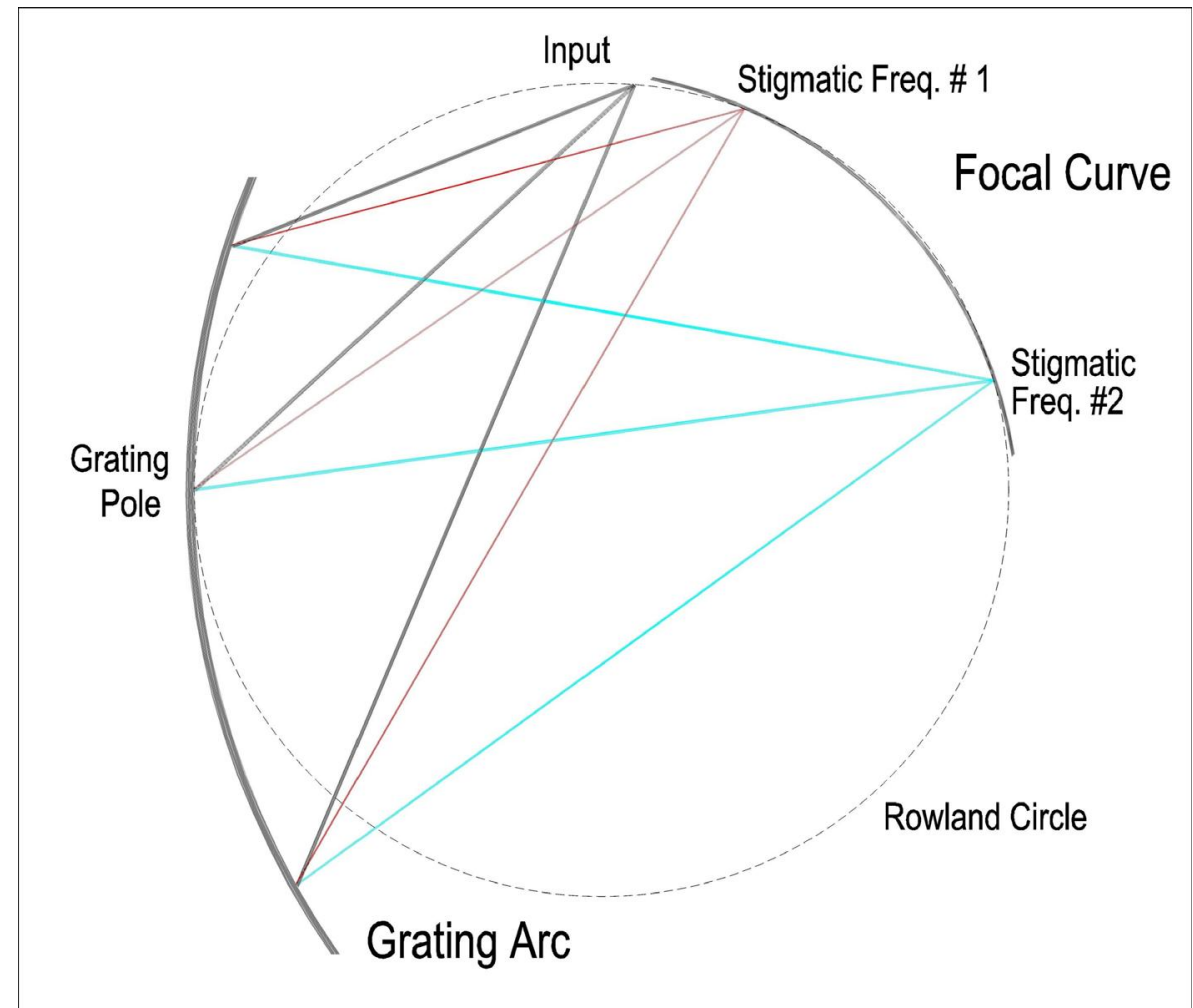

Figure 1. WAFIRs Design Schematic. Light from a single-mode feed illuminates the grating, which focuses and diffracts the radiation to a circular arc where detectors are located. The grating facets are positioned to provide perfect (stigmatic) performance at two frequencies on this detector arc.

\section{DESIGN OVERVIEW}

Z-Spec will initially be used on the $10.4 \mathrm{~m}$ CSO telescope in a single-beam, single-polarization configuration. It makes full use of the $1 \mathrm{~mm}$ atmospheric window. The designed resolving power varies from 400 at $1.54 \mathrm{~mm}$ to 250 at $0.97 \mathrm{~mm}$ for a grating with 480 facets. To achieve high sensitivity, $160 \mathrm{Si}_{3} \mathrm{~N}_{4}$ micromesh bolometers will be waveguidecoupled to the WaFIRS grating in Z-Spec, and the grating and detectors are cooled to $100 \mathrm{mK}$ to enable backgroundlimited performance. The cooling is provided by an adiabatic demagnetization refrigerator (ADR) in a standard liquid nitrogen and liquid helium dewar. The ADR is backed by a two-stage helium sorption refrigerator. Z-Spec receives light from the telescope with a corrugated feed horn, which tapers down to single-mode rectangular waveguide. The singlemode waveguide connects to the spectrometer input horn which gently tapers in both height and width. Light enters the parallel-plate waveguide via the input and illuminates the diffraction grating. The grating focuses and disperses light to a circular arc where rectangular waveguides receive the radiation and couple it to individually mounted bolometers. Thermal IR loading is eliminated with 4 low-pass metal mesh interference filters in the beam. Filters are mounted at the nitrogen, helium, and ${ }^{3} \mathrm{He}$ radiation shields, and directly on the opening of the corrugated feed itself. The filters have edges at $13 \mathrm{~cm}^{-1}, 12 \mathrm{~cm}^{-1}, 11 \mathrm{~cm}^{-1}$, and $10.3 \mathrm{~cm}^{-1}$, respectively. Frequencies below Z-Spec's band are naturally eliminated with the waveguide taper which couples the corrugated feed to the spectrometer feed. Z-Spec's bolometers are buffered with matched pairs of cryogenic J-FETs, and read-out at room temperature with a digital lock-in system under construction for Z-Spec, BICEP, and other non-multiplexed bolometer instruments. Initially, the single-beam spectrometer will chop on the sky, though eventually a second WaFIRS module could be added to double the on-source duty cycle or to observe both polarizations. 

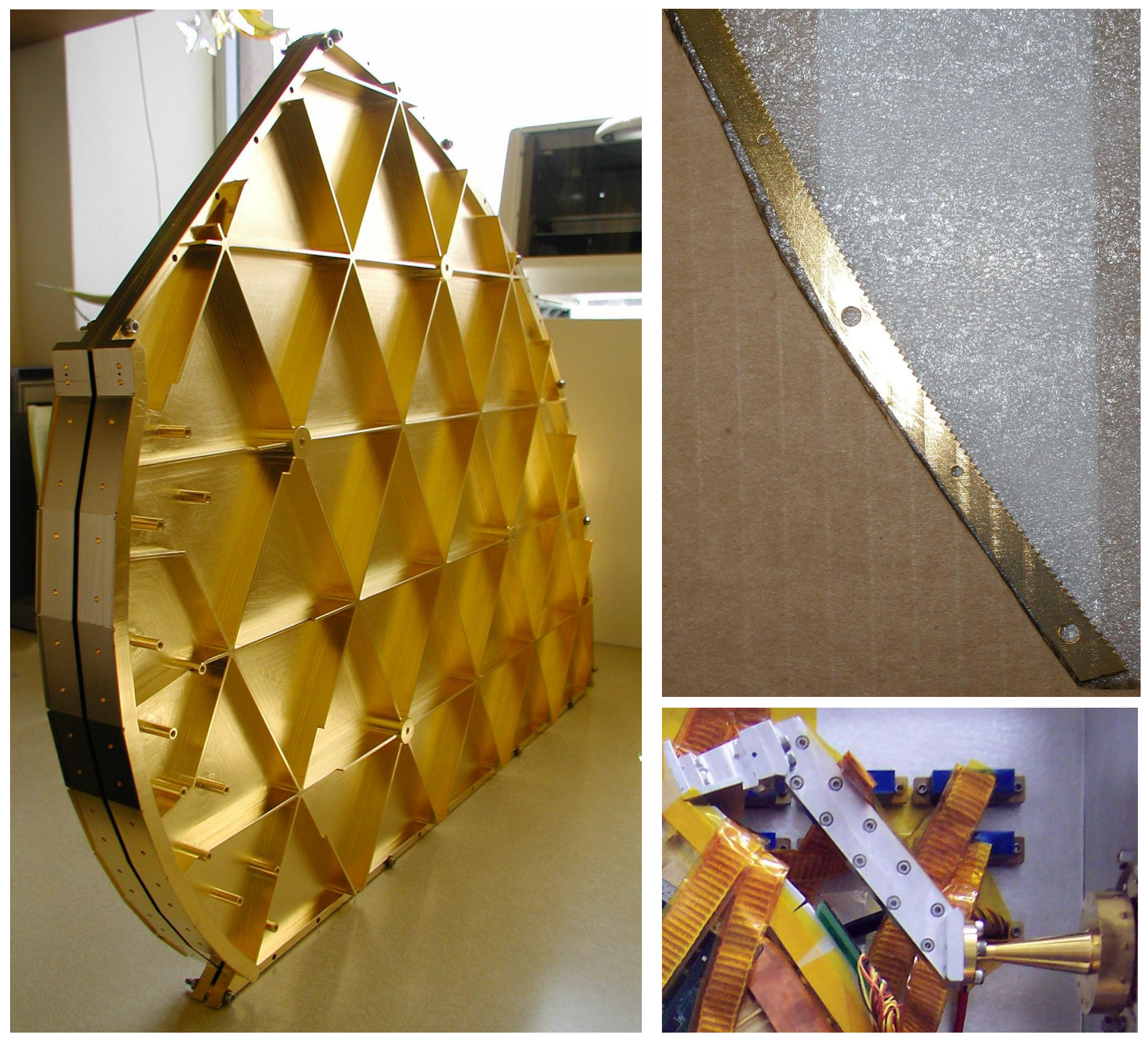

Figure 2. [Left] Z-Spec spectrometer assembled showing light-weighted rib structures. The input feed mounts on the top-most flat surface and bend blocks mount on the other eight flat surfaces. The longest dimension in the structure is $55 \mathrm{~cm}$. [Top Right] View of small segment of the grating. [Bottom Right] Corrugated feed horn for coupling to the telescope, waveguide taper, and input feed which illuminates the grating.

\section{CRYOGENIC WAVEGUIDE DIFFRACTION GRATING}

The design philosophy of the WaFIRS modules and our numerical approach are described in (B02), and the discussion here relies on this reference. Light propagates in the $\mathrm{TE}_{1}$ mode of parallel-plate waveguide, with two degrees of freedom. The grating design begins with a Rowland geometry, but then each facet of the grating is positioned such that the total path from the center of the input feed to the facet to the output position changes by exactly one wavelength as facet number is incremented. Solving this for two frequencies fixes the 2-D coordinates of each facet. For Z-Spec, the initial Rowland circle radius is $25 \mathrm{~cm}$, and the input position and stigmatic frequency output positions are selected on 
this circle. The grating then consists of 480 facets designed for stigmatic performance at these frequencies. The distribution of facets is not centered with respect to Rowland's vertex; 100 more lie on the side opposite the input position, as centering the large grating does not provide good illumination for the upper facets. Figure 1 illustrates these ideas. The blaze angle for all the facets is selected to be 27 degrees, based on published calculations and measurements of S-polarization blaze efficiencies. The parallel plate spacing is a compromise between the need to minimize waveguide propagation loss (wants larger spacing) and the need to avoid scattering into unwanted waveguide modes (wants smaller spacing). We chose $2.5 \mathrm{~mm}$ for our $190-310 \mathrm{GHz}$ system, which is overmoded by a factor of 3-5.

To implement the spectrometer, the two parallel plates, the grating, and the spacers were machined of 6061-T6 aluminum. Since the entire device is cooled to below $0.1 \mathrm{~K}$, mass is at a premium and the plates are designed with stiff, lightweight construction - a $1 \mathrm{~mm}$ thick web with $0.75 \mathrm{~mm}$ thick, $20 \sim \mathrm{mm}$ tall stiffening ribs arranged on a $50 \mathrm{~mm}$ triangular pattern. The operation of the spectrometer requires that the correct spacing be maintained across the entire region so that the same dispersion relation holds throughout. For Z-Spec the tolerance is of order 40 microns, and our finite element analysis on the plate structure confirmed that any gravity loadings produce deflections less than this value. The final polish, and the final flattening of the part after milling and stress relieving, were done by hand. The narrow grating piece is not self-supporting, and was carefully machined with a 5-axis mill while firmly supported. Thicknesses of the grating, and spacer pieces were carefully measured and maintained to within 10 microns. After machining, all parts were coated with 50 micro-inches of nickel, then a flash of gold. The thickness of nickel must be considered carefully, because the different CTE can cause the plates to bow upon cooling with the ribbed geometry. Photographs of the spectrometer module are shown in Figure 2.
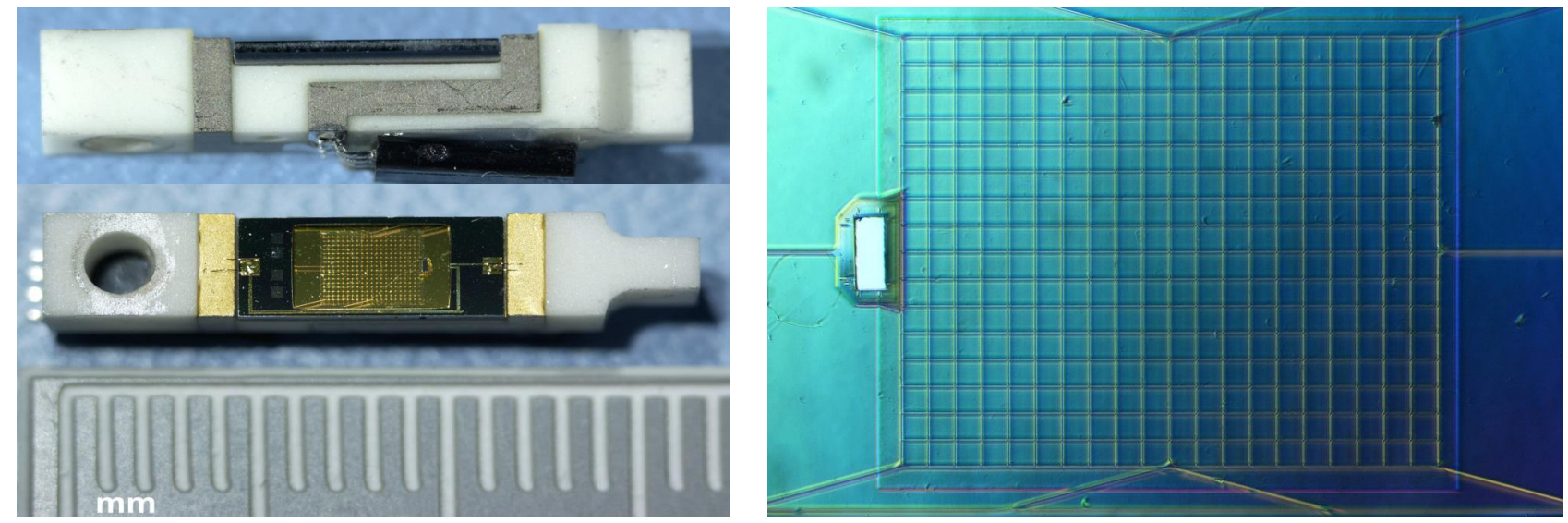

Figure 3. [Left] Alumina Z-Spec detector key with bolometer and backshort attached, with millimeter rule shown for scale. Each detector is individually mounted to allow easy exchange insuring perfect yield in the operating spectrometer. [Right] Zoomed view of the absorber mesh.

\section{BOLOMETERS AND COUPLING STRUCTURES}

Bolometers are thermal detectors limited by fundamental phonon fluctuations, with NEPs of $\sim\left(4 k T^{2} G\right)^{1 / 2}$, where $\mathrm{G}$ is the thermal conductivity of the supports and electrical leads. $\mathrm{G}$ is tuned for the expected optical loading and bath temperature by adjusting the amount of gold vapor-deposited on one of the $\mathrm{Si}_{3} \mathrm{~N}_{4}$ support legs. The target thermal conductance for the Z-Spec bolometers is $15 \mathrm{pW} / \mathrm{K}$ at $100 \mathrm{mK}$, tailored for the expected millimeter-wave background loading. This corresponds to a dark NEPs of $\sim 4 \times 10^{-18} \mathrm{~W} \mathrm{~Hz}^{-1 / 2}$ with a thermal time constants of $15 \mathrm{~ms}$. Our dark testing shows that these values have been achieved. The silicon nitride absorbers are etched from wafers 350 microns thick and attached to deep-trenched etched silicon backshorts. The thermistors are neutron-transmutation-doped (NTD)

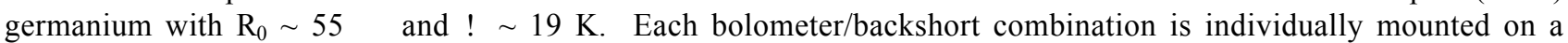
machined alumina module. One version of the alumina key is made for all the detectors, but several different quarterwave backshorts are needed to cover the full band with good efficiency. Figure 3 shows a Z-Spec detector, mounted with its backshort in the key. 
The detectors are mounted and illuminated in groups of 20 by waveguide bend blocks. The spectrometer focal curve is approximated with eight segments, each mounting one bend block. The blocks mount detectors above and below the plane of the parallel-plate waveguide, in an alternating fashion. This creates maximum space between physically adjacent bolometers to make room for readout wires and support framing, but requires no tapering or gaps between the individual waveguide feeds. The waveguide feeds have constant rectangular cross section ( $2.5 \mathrm{~mm} \times 1.57-$ $1.95 \mathrm{~mm}$ ) and use curved bends with an $8.25 \mathrm{~mm}$ radius. Curved bends have significantly better transmission than mitred bends, particularly for higher order modes and are easier to directly machine. The extra length beyond the focal curve required by curved bends is mitigated somewhat by having a partial S-shaped bend as seen in Figure 4[Left]. The rectangular waveguide feeds can support several modes and so power is mixed between modes significantly by the bends. However, the bolometer absorption should be relatively independent of the illuminating waveguide mode.

The first bend block, fabricated by Custom Microwave, is shown in Figure 4 [Center]. The blocks are fabricated by bolting 21 slices together. Most of the slices are copies of one of two primary types, each machined to produce two waveguide halves. There are two special end slices which have only one waveguide half machined into one side. The slices are assembled with captive alignment pins that match up the waveguide halves in adjacent slices. The stack of slices is further machined and gold plated to complete the block. The other seven blocks are currently being machined and are scheduled to be completed by the end of August 2004.
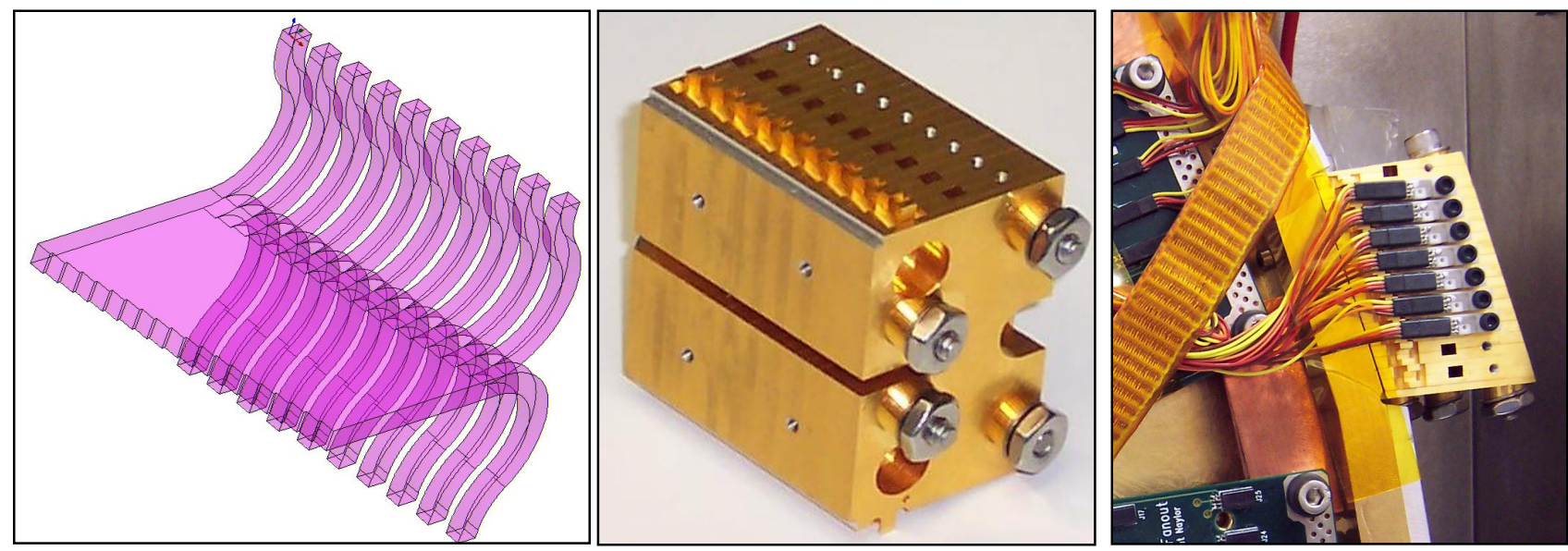

Figure 4. Z-Spec bend blocks. Light from the parallel plate region is coupled to detectors via waveguide bends. [Left] concept - shows alternating geometry. [Center] Complete block built up of 21 nearly identical machined slices. [Right] Block on the spectrometer with 7 detector keys mounted.

\section{CRYOGENIC DESIGN}

Reaching the background limit for a millimeter-wave spectrometer requires detectors cooled to $\mathrm{T} \sim 0.1 \mathrm{~K}$. Furthermore, the optical power incident on a detector from inside the instrument should be small relative to the in-band background power from the sky and telescope. For a detector coupling a fractional bandwidth of $1 / 300$, the loading from the sky and telescope in good weather at the CSO is $0.2-0.4 \mathrm{pW}$. For comparison, the power coupled by a $2 \mathrm{~mm}$ square detector in a $2 \mathrm{~K}$ enclosure is $4 \mathrm{pW}$, hence careful attention must be paid to cryogenics and filtering. In Z-Spec, there are no band-limiting filter elements downstream once the light enters the spectrometer, so the entire spectrometer must be cooled below $1 \mathrm{~K}$.

Z-Spec's cryogenic system allows for efficient cooling of the spectrometer and detectors from room temperature through 2 gas-gap heat switches (Figure 5). Gas-gap switches provide much higher on-stage conduction than mechanical switches; a necessity due to the large $100 \mathrm{mK}$ mass $(4 \mathrm{~kg}$ of aluminum). We use an adiabatic demagnetization refrigerator (ADR) to achieve the sub- $100 \mathrm{mK}$ base temperature Our system uses a ferric ammonium alum (FAA) salt pill for which the entropy is entirely magnetic below about $1.5 \mathrm{~K}$. Upon removing the magnetic field, the entropy available for cooling is approximately $\mathrm{R} \ln 6$ per mole of salt, or $7 \mathrm{~J} / \mathrm{K}$ with our 0.5 mole pill; the entropy of 
the grating at $1.5 \mathrm{~K}$ is only $0.5 \mathrm{~J} / \mathrm{K}$. For this reason, we cool the entire spectrometer with the ADR, rather than engineering a thermal break between the spectrometer plates and the detector modules.
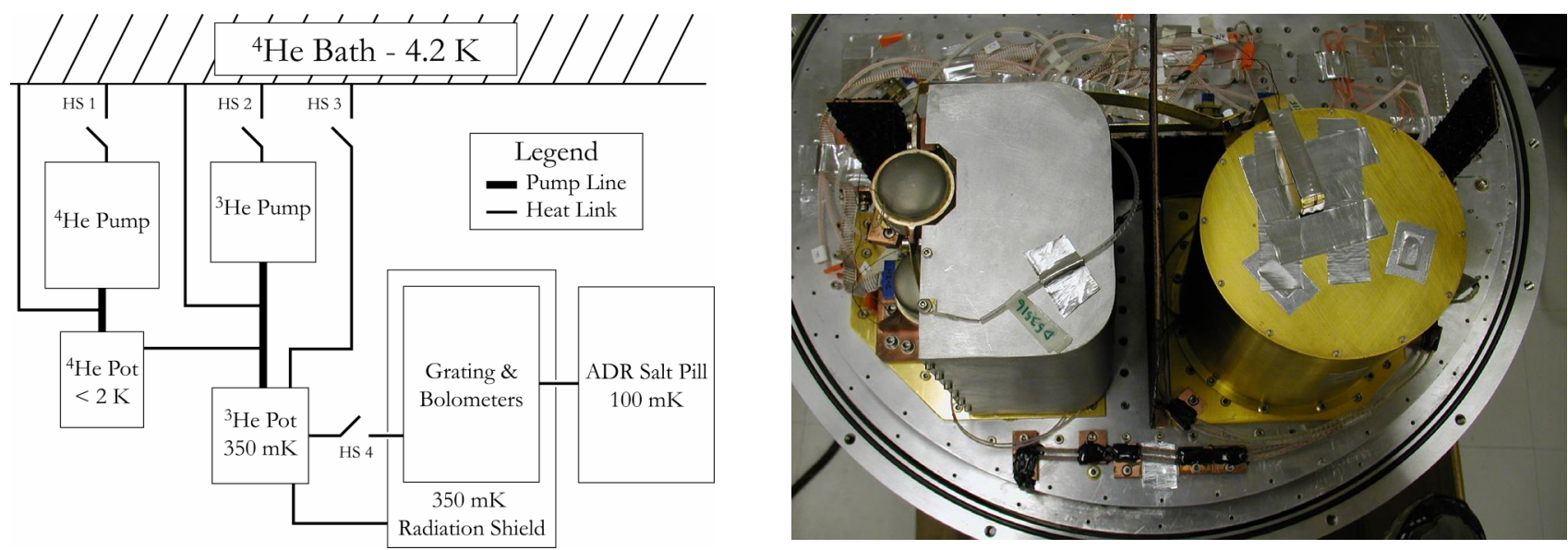

Figure 5: Z-Spec cryogenic design. The spectrometer and bolometers are cooled by an ADR and ${ }^{3} \mathrm{He} /$ ${ }^{4} \mathrm{He}$ sorption refrigerator. The fridge is cycled in stages. First, a magnetic field is applied to the ADR, and HS's $3 \& 4$ are closed to conduct the heat of magnetization to $4 \mathrm{~K}$. Then ${ }^{4} \mathrm{He}$ is condensed by opening HS 1 and heating the ${ }^{4} \mathrm{He}$ pump with a resistive heater. Liquid ${ }^{4} \mathrm{He}$ condenses in the pump tube at the thermal link and drips into the evaporator. Heat switch 1 (HS1) is closed to cool the pump, which pumps the liquid ${ }^{4} \mathrm{He}$ in the ${ }^{4} \mathrm{He}$ evaporator to $<2 \mathrm{~K}$. The ${ }^{3} \mathrm{He}$ stage is then cycled by opening HS 3 , opening HS 2, and heating the ${ }^{3} \mathrm{He}$ pump. The ${ }^{4} \mathrm{He}$ stage condenses liquid ${ }^{3} \mathrm{He}$ at $\sim 2 \mathrm{~K}$, cooling the ADR to $2 \mathrm{~K}$ and exhausting the ${ }^{4} \mathrm{He}$ in the process. The ${ }^{3} \mathrm{He}$ pump is then cooled by closing HS 2 , cooling the ${ }^{3} \mathrm{He}$ evaporator to $400 \mathrm{mK}$. During the cooldown to $400 \mathrm{mK}$, HS 4 is opened and the ADR is demagnetized to $100 \mathrm{mK}$. The exact timing of the ADR cycle depends on the dissipation of HS 4, the thermal time constant of the ADR and spectrometer, and is being determined empirically.

$\mathrm{A}^{3} \mathrm{He}$ system is used as a $0.4 \mathrm{~K}$ thermal guard, intercepting the heat conducted through the wires, and providing a radiation shield around the large spectrometer surface area to eliminate loading from the $4 \mathrm{~K}$ radiation. In Z-Spec, the ${ }^{3} \mathrm{He}$ stage is integrated with a closed cycle ${ }^{4} \mathrm{He}$ stage, which provides the condensation temperature required for the ${ }^{3} \mathrm{He}$, and is used to pre-cool the salt pill and cold stage down to $\sim 1 \mathrm{~K}$ before demagnetization. This closed-cycle ${ }^{3} \mathrm{He} /{ }^{4} \mathrm{He}$ combination refrigerator is completely modular, it requires no pumping on the main cryostat bath, and its operation is fully automated. The grating cools to below $100 \mathrm{mK}$, and has demonstrated a hold time of 20 hours. A schematic and images of some components of Z-Spec's cryogenic system are shown in Figure 5.

\section{SPECTROMETER TESTING}

Our initial testing of the spectrometer was performed warm, using a sweepable mm-wave source and a diode detector in single-mode waveguide. A few of the representative spectra are shown in Figure 8. The system performed well over the entire band, demonstrating substantial improvement over our first prototype. The spectra show $\sim 30 \%$ transmission and resolving power very close to that predicted for each frequency. The performance of the first bend block was measured independently as part of the measurement, and was found to have better than $95 \%$ transmission, when the mode conversion is taken into account. The warm single-mode measurements suffer from some standing waves, or multiple reflections as the detectors and sources are not well matched.

With the successful integration of the cryogenic system, and the fabrication of the first bolometers, it was possible to make the first cryogenic measurements at the frequency of the first available bend block, around $225 \mathrm{GHz}$. These measurement made use of the same sweepable mm-wave source as the warm measurements, but this time coupled through free space and attenuated by 8-10 orders of magnitude to avoid saturating the detectors. The profiles for the 11 

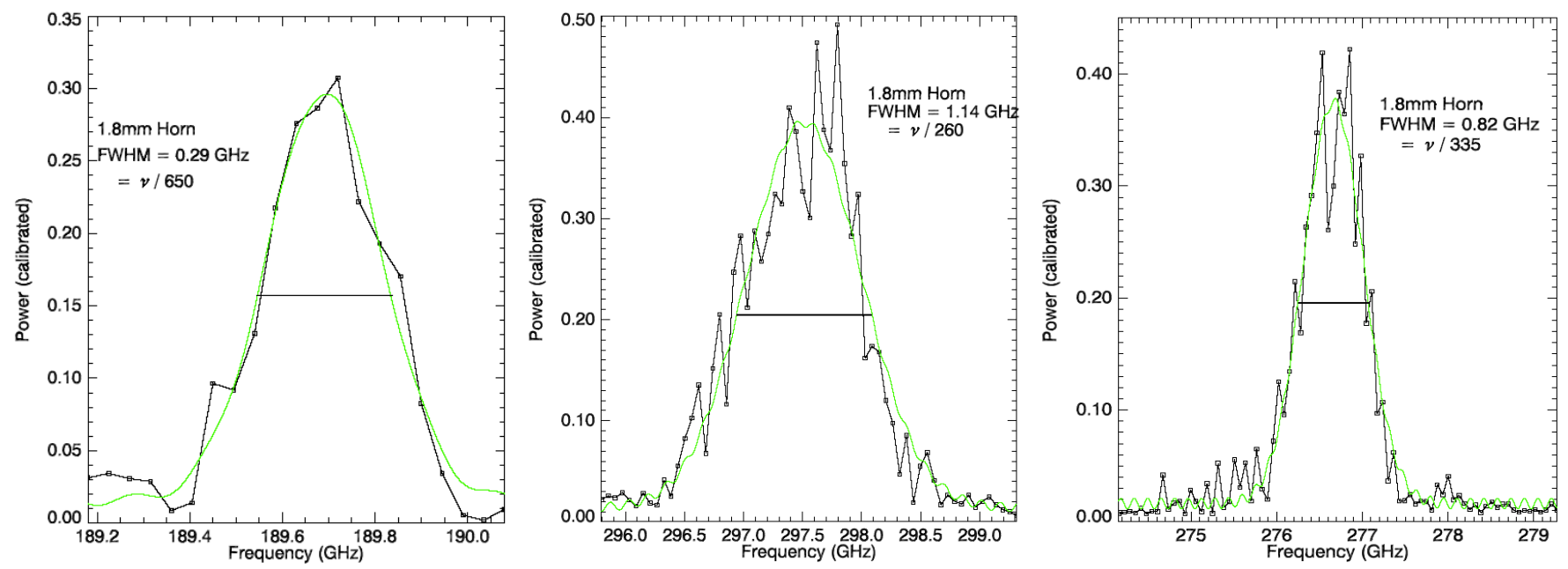

Figure 6: Z-Spec spectrometer warm measurements. The profiles were obtained with a sweepable mm-wave source and a single-mode waveguide-coupled diode detector, and the coupling on the vertical axis is the measured ratio between the source-detector chain with and without the spectrometer. Resolving powers are comparable to those predicted with the numerical model.

operational bolometers are plotted it Figure 9, with Gaussian fits to each profile. The measured profiles represent the convolution of the intrinsic resolving power of the spectrometer with the width of the detector waveguide feed. Our scalar model suggest an intrinsic resolving power of 1 part in 380 , and the detector feed width corresponds to 1 part in 330 at $225 \mathrm{GHz}$. We note that modeling the details of this focal curve coupling requires a complete vector solution of the field equations in the spectrometer, which is beyond the scope of our current numerical model. The Gaussian fits to the data show FWHM between $1 / 330$ and $1 / 215$, which is already encouraging. These fits reflect larger widths than the main lobe of the profile due to the presence of a substantial side lobe on the high-frequency side of each profile. Because this was not a feature of the warm measurements, it is the result either some aspect of the bend-block / bolometer coupling, or more likely, a loss of coherence due to deformation of the parallel plates under cooling. In any case, large difference in the profiles from channel to channel are not likely to be real, and the channel-to-channel difference may be due to some systematic uncertainty in our measurement process. As of this writing, we are in the process of confirming these data with more sensitive broadband measurements.

Because of the 8-10 orders of magnitude by which the monochromatic source is attenuated for these measurements, the absolute coupling through the system cannot be estimated with spectral profiles alone. The couplings plotted in Figure 9 are determined as follows: Each profile integrates to determine a measured equivalent square bandwidth, referred to the peak value. This bandwidth is used to calculate the loading due to ambient and $77 \mathrm{~K}$ loads, assuming full coupling in a single spatial / polarization mode. This is then compared with the measured loading difference, easily deduced from the bolometer I-V curves (or load curves). The result is that the peak couplings are between 38\% and 63\%. Because we have not yet measured the full broadband response of the system, it is possible that the system has high-frequency leaks, which would increase the measured loading with out-of-band power, and correspond to a smaller in-band coupling. We are optimistic however, as 1) the filter technology is very mature, and substantial experience now shows that high-frequency leaks with the metal-mesh filter stacks are rare, 2) the available $\mathrm{A} \square$ product for thermal IR photons through the waveguide feed and into the spectrometer is small and further diluted for any individual detector because of the geometry of the grating and 3) the multi-mode bolometer measurements are broadly consistent with the $30-40 \%$ measured with the single mode measurements. The coupling we measure is close to what is expected based on the known sources of loss, listed in Table 1. 


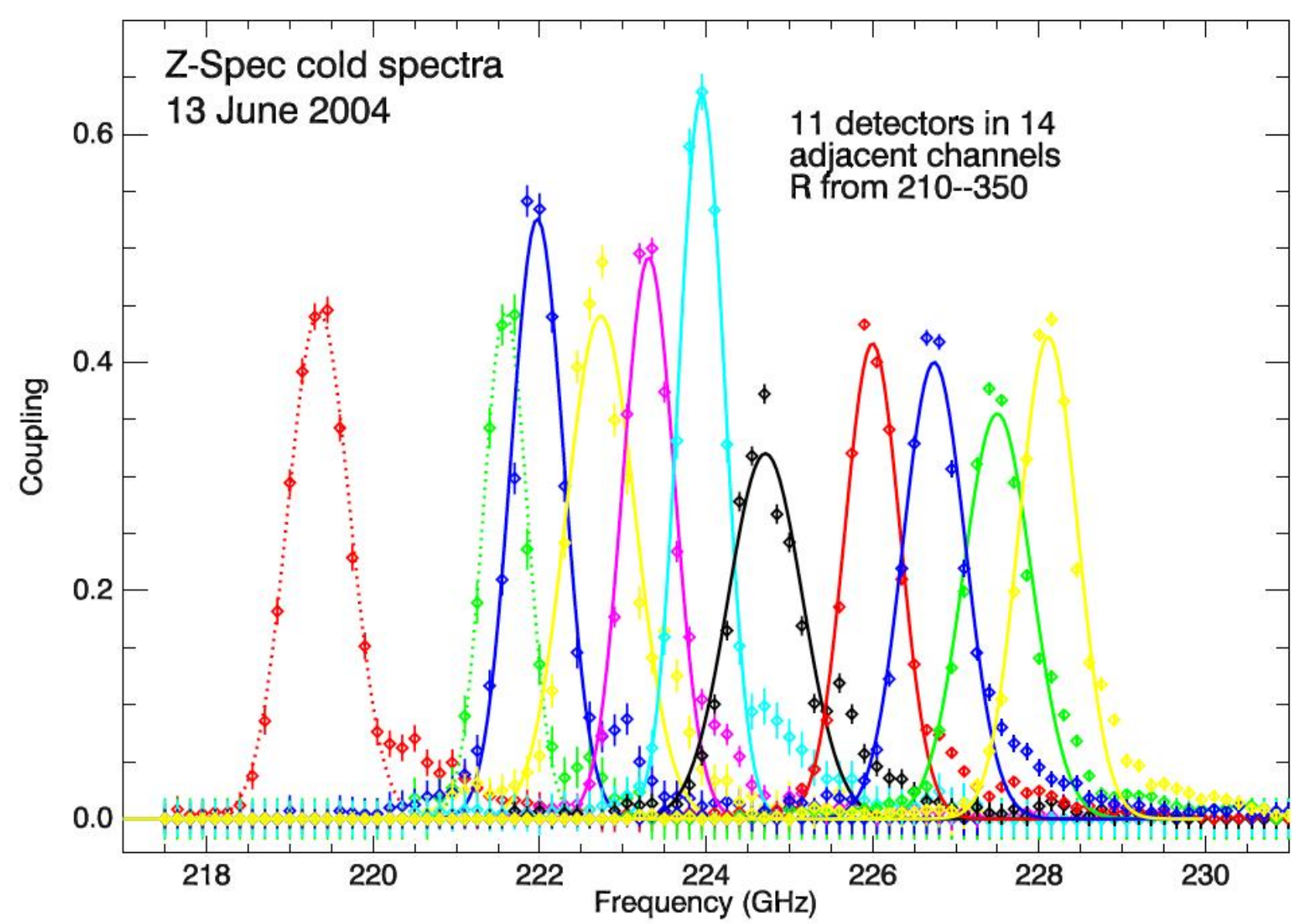

Figure 7: Z-Spec spectrometer first cryogenic measurements. The profiles were obtained with a sweepable mm-wave source radiating via a horn outside the cryostat, chopped with a blade. 11 detectors were installed on the first bend block, each detector's profile was measured independently. The plotted coupling is based on the measured loading with ambient and $77 \mathrm{~K}$ loads, as described in the text.

Table 1. Z-Spec throughput estimates

\begin{tabular}{|c|c|}
\hline Mechanism & Coupling \\
\hline IR blocking filters & $0.90-0.94$ \\
\hline Grating illumination & $0.79-0.89$ \\
\hline Blaze efficiency & $0.95-1.0$ \\
\hline WG propagation loss & 1.0 (cold-calc) 0.95 (warm-calc.) \\
\hline Detector + backshort & 0.85 (estimated) \\
\hline Product & $\mathbf{0 . 5 5 - 0 . 7 0}$ \\
\hline
\end{tabular}

Another source of loss that is very difficult to estimate is any imperfections in the of the five waveguide flanges throughout the system. In summary, the measurements thus far suggest that Z-Spec will provide and unprecedented combination of bandwidth and throughput, and we look forward to demonstrating this new instrument on the telescope within a year. 


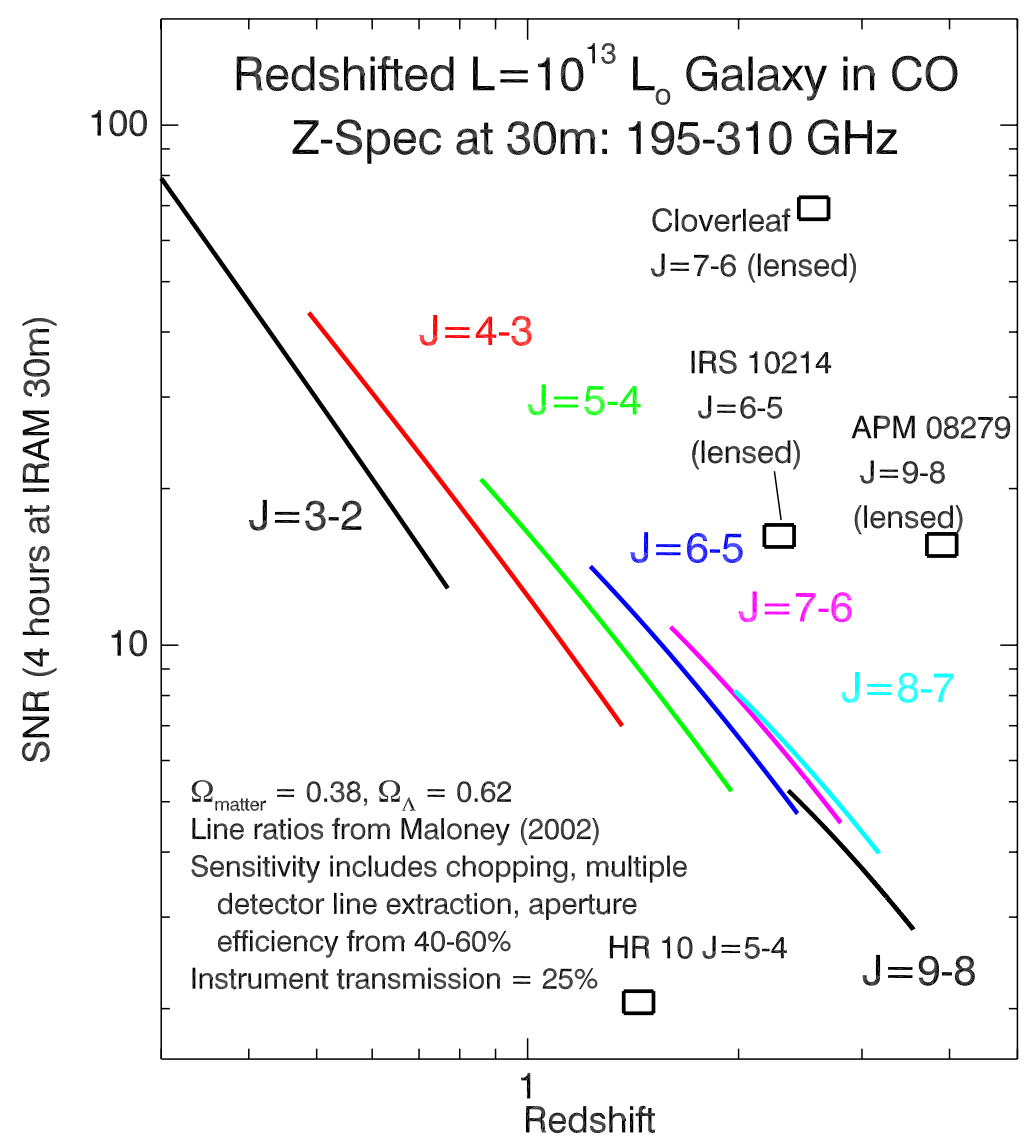

Figure 8: Z-Spec sensitivity to an $\mathrm{L}=10^{13} \mathrm{~L}_{\text {solar }}$ galaxy at the IRAM 30-meter telescope. Apart from a small gap from $0.8<\mathrm{z}<0.9$, two or more CO lines are always redshifted into Z-Spec's band. In good weather, these bright submillimeter galaxies can be detected in their CO lines at a rate of about 2 per night.

\section{GROUND BASED ASTRONOMICAL CAPABILITIES}

With sensitive $100 \mathrm{mK}$ bolometers and high throughput, Z-Spec will operate near the fundamental limit set by the fluctuations of the sky and telescope background, as described in N02. The detector sensitivity is $4 \times 10^{-18} \mathrm{~W} \mathrm{~Hz}^{-1 / 2}$, and the background noise under good conditions will be $1 \times 10^{-17} \mathrm{~W} \mathrm{~Hz}^{-1 / 2}$, increasing somewhat with frequency.

At the CSO, an integration time of 4 hours provides a $1 \square$ sensitivity of $5 \times 10^{-20} \mathrm{~W} / \mathrm{m}^{2}$, while the much larger IRAM telescope allows integration to sensitivities of $1 \times 10^{-20} \mathrm{~W} / \mathrm{m}^{2}$ in the same time under the best weather conditions. These sensitivities are sufficient to measure $\mathrm{CO}$ lines in the brightest submillimeter galaxies. Figure 8 plots the sensitivity of Z-Spec to a bright submillimeter galaxy as a function of redshift at the IRAM 30-meter. The CO line luminosities are estimated as fractions of the total source based on a model by Maloney et al. (personal communication); the fractions vary from $4 \mathrm{e}-6$ to $5 \mathrm{e}-5$. The plots show each CO line appearing in the 190-310 GHz band in turn as redshift increases. At least two lines are observable for all redshifts greater than 0.9 , which provides an unambiguous redshift measure. For $\mathrm{z}<0.9$, one or two candidate redshifts can be obtained from a single CO line. 


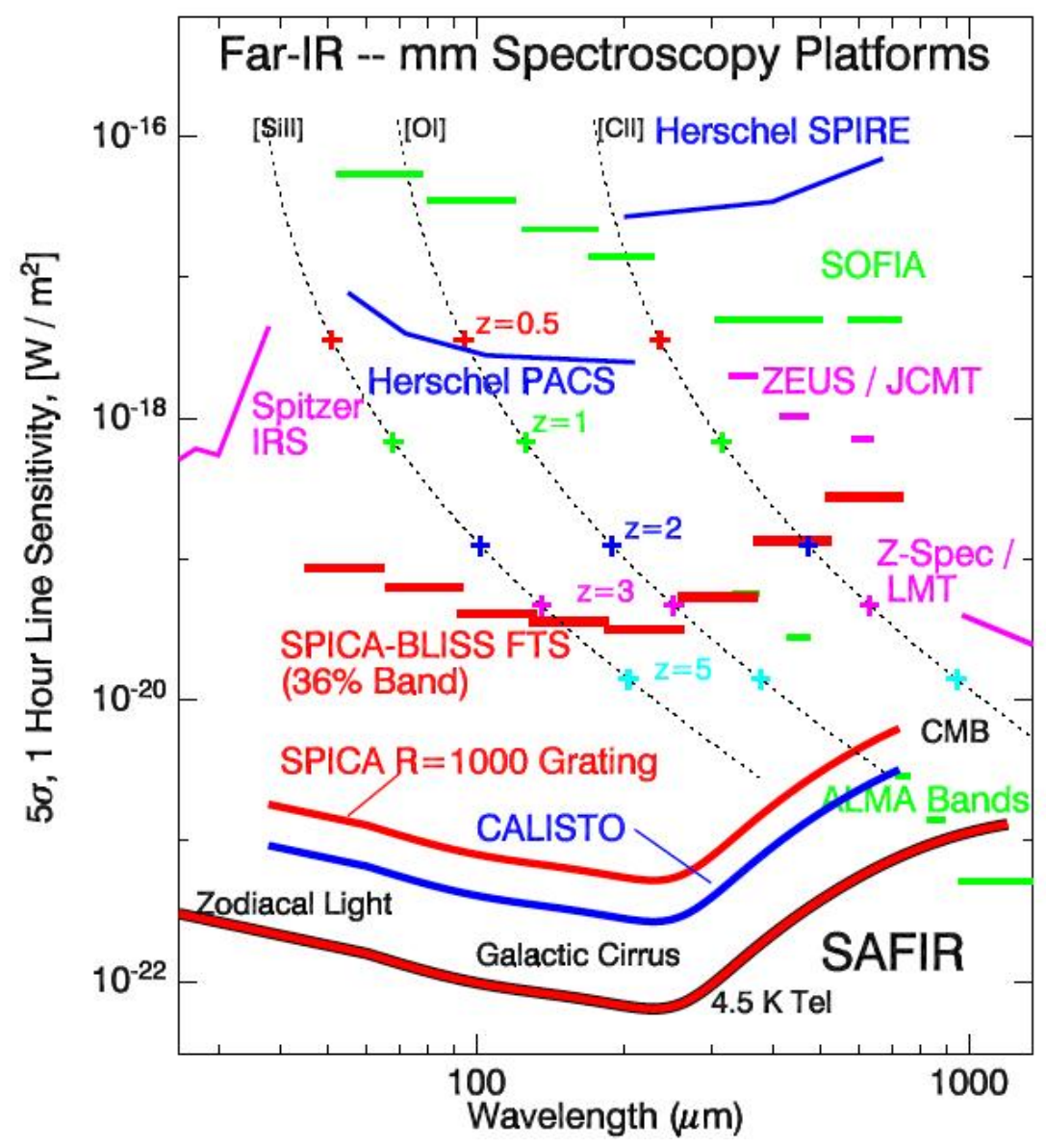

Figure 8: Sensitivity of far-IR spectroscopy platforms. Values for SOFIA, Herschel, Spitzer, and ALMA are taken from the instrument web pages. For ALMA, we assume a $300 \mathrm{~km} / \mathrm{s}$ linewidth. Values for the ground-based ZEUS and Z-Spec instruments are taken from published values. BLISS stands for Background-Limited IR-Submillimeter Spectrograph, and curves are plotted for a dispersive grating system on three potential cryogenic platforms: 1) the Single Aperture Far-Infrared observatory SAFIR, a 10-meter $4.5 \mathrm{~K}$ observatory under study as part of NASA's Vision Missions program, 2) the Cryogenic Aperture Large Infrared Space Telescope Observatory (CALISTO), a concept for a 4x6 meter off-axis aperture, and 3) SPICA, the Japanese Space Infrared Telescope for Cosmology and Astrophysics, a 3.5 meter $4.5 \mathrm{~K}$ telescope tentatively scheduled for launch in 2012. Also, the sensitivity of a Fourier-transform device is also plotted for SPICA. For these cold telescopes, the sensitivity is limited by photon noise from the astrophysical backgrounds. The calculations assume $75 \%$ aperture efficiency and $25 \%$ total instrument transmission. Overplotted are spectral line intensities from ULIRG at various redshifts assuming a fractional line intensity of $10^{-3}$, and the current cosmological model $\left(\square_{\mathrm{vac}}=0.73, \square_{\mathrm{mat}}=0.27, \mathrm{H}_{\mathrm{o}}=71\right)$.

\section{POTENTIAL OF WAFIRS-ARCHITECTURE ON A COLD SPACE TELESCOPE}

One of the most exciting aspects of the new WaFIRS technology is the potential for sensitive broad-band far-IR spectroscopy when combined with a cryogenic space telescope. Far-IR spectroscopy is the key to studying the properties of the dusty galaxies as they are resolved from the background with the continuum instruments on Spitzer, Herschel, and the new ground-based facilities. The wide variety of spectral features in mid- and far-IR are extinction- 
free probes of the conditions in all phases of the interstellar medium: ionized, neutral atomic, and molecular. In contrast, optical and near-IR feature generally probe only the low extinction surfaces of dense regions, which can represent only a small portion of the entire galaxy. For sources which are completely obscured optically, the far-IR line suite can also be used as a redshift measure. Unfortunately, these measurements will not be possible with Herschel and SOFIA -- their spectrometers are not capable of probing beyond $\mathrm{z} \sim 1$ due to the photon background from the warm optics. In Figure 9 , we plot the dramatic gains possible in the far-IR with a dispersive direct-detection spectrograph on a cryogenic space telescope. With even a 3-meter class cold telescope, such an instrument would enable measurements of spectral lines in bright galaxies to beyond $\mathrm{z} \sim 5$.

Table 2. Scalability of WaFIRS Modules

\begin{tabular}{|c|c|c|c|c|}
\hline \multicolumn{2}{|c|}{ Design Parameter } & Z-Spec (built) & SPICA far-IR & SPICA far-IR \\
\hline \hline Wavelength & $\mu \mathrm{m}$ & $970-1540$ & $160-300$ & $160-300$ \\
\hline Medium & & Vacuum & Vacuum & Silicon \\
\hline Detectors & $\#$ & 160 & 500 & 500 \\
\hline Facets & $\#$ & 480 & 4000 & 4000 \\
\hline Resolving Power & $/$ & $250-400$ & $1000-1600$ & $1000-1600$ \\
\hline Spacing & $\mathrm{mm}$ & 2.5 & 0.6 & 0.18 \\
\hline Tolerance & $\mu \mathrm{m}$ & 40 & 5 & 1.5 \\
\hline Length & $\mathrm{mm}$ & 610 & 550 & 160 \\
\hline Efficiency & & $0.78-0.85$ & $0.90-0.93$ & $0.90-0.93$ \\
\hline
\end{tabular}

WaFIRS is an excellent candidate for such an instrument, given its high throughput, broad bandwidth, and compact size. The architecture is scalable to shorter wavelengths, and higher resolving powers, as indicated in Table 2. We emphasize the advantages of the 2-D architecture - the potential for stacking multiple modules, and the potential for dielectric immersion making the modules even smaller.

\section{CONCLUSION}

We present the first testing of our broad-band millimeter-wave spectrometer Z-Spec. Z-Spec is based on a waveguide grating architecture WaFIRS, and is designed to couple the entire 190-310 GHz atmospheric window simultaneously. Warm testing demonstrates that the waveguide grating has 30-40\% efficiency, and performs well over the entire band. The first cold testing with bolometeres at $225 \mathrm{GHz}$ shows a resolving power of $200-350$, close to what is expected, and loading measurements are consistent with a total throughput of 30-40\%. Further measurements are in progress to confirm these results, and we are completing the construction of Z-Spec in preparation for the first astronomical measurements in early 2005. The success of this first WaFIRS prototype makes highlights its potential for the future of far-IR / submillimeter astrophysics -- sensitive far-IR spectrographs on cold space telescopes.

\section{ACKNOWLEDGEMENTS}

We than Frank Rice (Caltech) for the use of his mm-wave multiplier chain, and Warren Holmes (JPL) for his work in testing Z-Spec detectors.

\section{REFERENCES}

[B02] Bradford, C.M. et al., "WaFIRS: a waveguide far-IR spectrometer for spaceborne astrophysics," Proc SPIE, 4850-162. 2002

[N02] Naylor, B.J. et al., "Z-Spec: a broadband, direct-detection millimeter-wave spectrometer," Proc SPIE, 4855-239. 2002 